\title{
Spin-dependent current in silicon p-n junction diodes
}

\author{
O.V. Tretyak, O.I. Kozonushchenko, K.V. Krivokhizha, A.S. Revenko \\ Taras Shevchenko Kyiv National University, Radiophysics Department, \\ 64, Volodymyrs'ka str., 01601 Kyiv, Ukraine
}

\begin{abstract}
We have used electrically detected spin-dependent paramagnetic resonance to investigate the non-equilibrium conductivity in a silicon diode. In order to create paramagnetic centers, we used diode with a polished surface (that includes $p-n$ junction). The dependence of relative changes in the amplitude of a signal under resonance conditions and the total value of current through the diode were investigated. We have found the presence of inversion channel on the surface of $p-n$ junction and proposed the model of the influence of spin resonance on the channel conductivity. The upper value of the time constant inherent to the spin-dependent process was determined as approximately $10^{-6} \mathrm{~s}$. The influence of the spin-dependent process on the charge state in inversion channel has been discussed.
\end{abstract}

Keywords: EDMR, ESR, polished surface, paramagnetic states, inversion layer.

Manuscript received 13.10.09; accepted for publication 22.10.09; published online 30.12.09.

Non-equilibrium conductivity can depend on the spin orientation of free carriers and charges in localized states in the bandgap. Various experiments reported about spin-dependent scattering [1], spin-dependent recombination [2-4], spin-dependent hopping conductivity [5]. Generally, the spin dependence of current was observed via its changes under the conditions of spin resonance. The theory of the spindependent non-equilibrium conductivity under spin resonance conditions was developed in [6].

The first report of a spin-dependent recombination in mechanically treated silicon was presented in [2]. More appropriate object for spin-dependent recombination in silicon is a diode with mechanically treated surface, which includes $p$ - $n$ junction (Fig. 1).

A detailed juxtaposition of experimental data and the theory of spin-dependent recombination (SDR) is difficult, which is caused by the following reasons.

Up to now, in all works devoted to SDR investigations the change of non-equilibrium conductivity $\Delta \sigma_{s}$ under spin resonance was always compared with the total conductivity $\sigma$; in this paper we operate with current since $\left(\frac{\Delta \sigma_{s}}{\sigma}=\frac{\Delta I_{s}}{I_{\Sigma}}\right)$. The total current $I_{\Sigma}$ consists of spin-dependent $\left(I_{s}\right)$ and spinindependent $\left(I_{n s}\right)$ parts: $I_{\Sigma}=I_{s}+I_{n s}$. As a rule, $I_{s}<<I_{n s}$, so an error can reach a value higher than one order.
Moreover, the dependence of $\frac{\Delta I_{s}}{I_{\Sigma}}$ on temperature or applied voltage may not represent the real process in samples (in the case of using $I_{\Sigma}$ instead of $I_{s}$ as denominator in $\left.\frac{\Delta I_{s}}{I}\right)$. Therefore, the information about the total spin-dependent current (that should be used as denominator) is a question of principle importance.

In this work, we present the attempt to estimate (being based on experimental data) the value of $I_{s}$ and to ascertain its mechanism in a silicon diode D-242 with polished surface of $p-n$ junction.

In silicon power diodes, the reverse current is defined by generation-recombination mechanism $\left(I \sim \frac{1}{\tau}, \tau-\right.$ time constant $)$. After mechanical treatment on the surface of diode, new channels of carrier transportation are created, including the spin-dependent one, $I_{\Sigma}=I_{0}+I_{s}+I_{n s}$, where $I_{0}$ is the current of unpolished sample; $I_{n s}$ and $I_{s}$ are spin-independent current and spindependent current, respectively (the latter appears after mechanical treatment of the diode). Each of these currents is inversely proportional to $\tau$; so it is possible to assume that determining the value of correspondent time constants $\tau_{0}, \tau_{n s}, \tau_{s}$ would lead to the possibility to define the ratio $\Delta I_{s} / I$ in a more accurate way. 


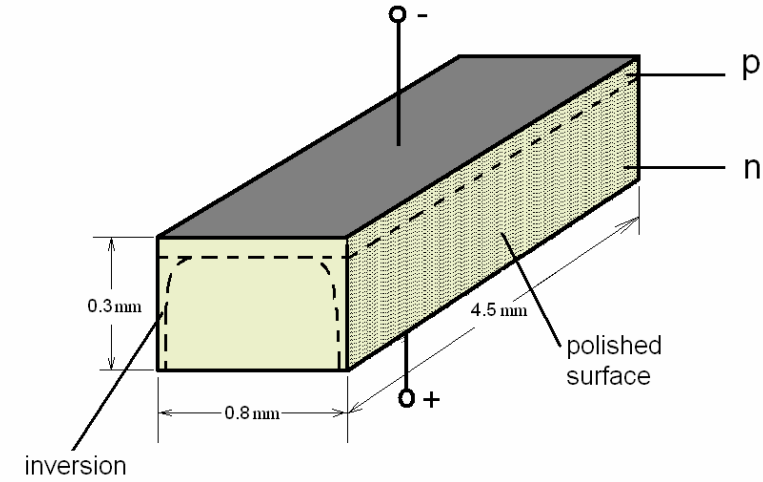

Fig. 1. Diode with polished sides.

In Fig. 1 the schematic image of diode is shown. Two sides are polished with diamond pasta with the granular size of 3 to $5 \mu \mathrm{m}$. The experimental currentvoltage characteristics of the diode before and after polishing are shown in Fig. 2. Before polishing, the reverse current-voltage characteristic was in a good correlation with the corresponding formulae for generation-recombination mechanism of current (Fig. 2, curve 4):

$$
j(U)=\frac{\text { end }}{\tau}\left(e^{e U / k T}-1\right),
$$

where $n$ is the equilibrium concentration of carriers, $d-$ width of space charge region, $\tau-$ time constant.

We have obtained the lifetime value for minority carriers $\tau$ in the diode gained from the transient characteristic [7]: it is about $10^{-5} \mathrm{~s}$. After polishing, the reverse current increased significantly. Also, after polishing the spin-dependent current in the diode was observed under spin resonance conditions (and wasn't observed before polishing).

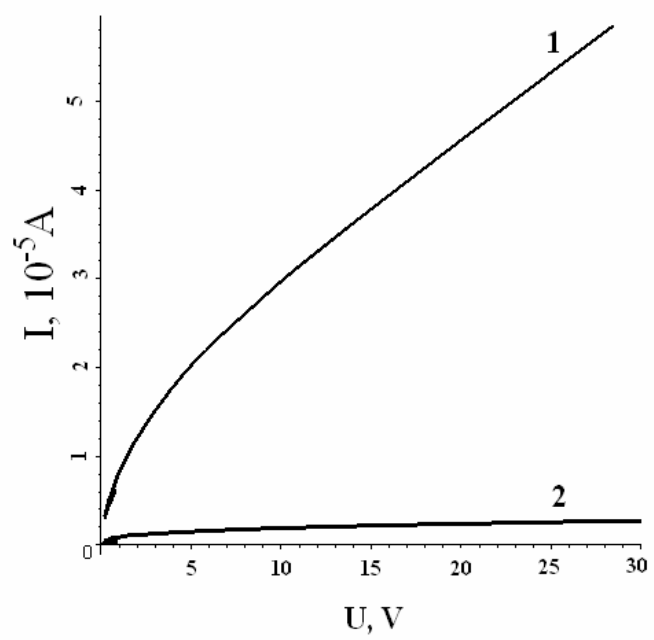

Fig. 2. Current-voltage characteristics (CVC) for the diodes: 1 - polished and 2 - untreated.

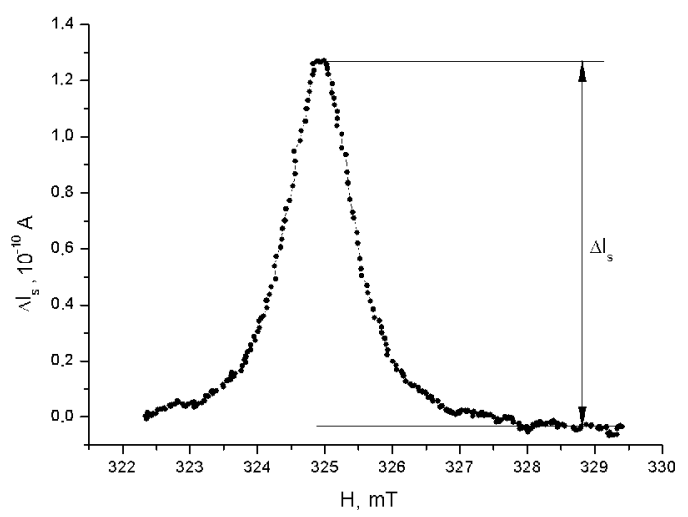

Fig. 3. Typical spectrum of EDMR.

The method of measuring the changes in conductivity of minority carriers under spin resonance is well known and presented, for example, in [2]. The typical curve of $\Delta I_{s}(H)$ is shown in Fig. 3. The maximum of $\Delta I_{s} / I_{\Sigma}$ was about $10^{-6}$.

In Fig. 4 the dependence of $\Delta I_{s}$ on the modulation frequency of the UHF at reverse voltage $15 \mathrm{~V}$ is shown. In theory, the time constant of spin-dependent current $\tau_{s}$ can be determined ([8]) from this dependence using the formula

$\Delta I\left(f_{\text {mod }}\right)=\Delta I_{0}$ th $\left(\frac{1}{4 \tau_{s} f_{\text {mod }}}\right)$,

where $\Delta I_{0}$ is the amplitude at low frequencies. But in our case, the value of $\tau_{s}$ cannot be determined because of hardware limitations. It is clear from Fig. 4 that the time constant of spin-dependent current $\tau_{s}$ is less than $10^{-6} \mathrm{~s}$.

All the aforementioned is the evidence of the following assumption: both the increase of the reverse current (due to polishing) and appearance of the spindependent current are results not only of creation of additional generation-recombination pairs, but also of the creation of a new conductivity channel in subsurface layers.

It is known from literature data $[9,10]$ that mechanical treatment of the surface of silicon leads to creation of an inversion layer. The increasing value of reverse current, the absence of $\mathrm{CVC}$ saturation for the polished diode (Fig. 2), the sign of changes in the reverse current under spin resonance (increasing) and minimum value of $\tau_{s}<10^{-6}-$ all these aspects allow to assert that the inversion layer of conductivity is created in our case.

It is clear that for voltages higher than $\approx 15 \mathrm{~V}$ the reverse current is determined mainly by conductivity of the inversion channel that typically has ohmic characteristics. For voltages lower than $\approx 15 \mathrm{~V}$, the generation-recombination mechanism is the main one in transporting carriers through the $p-n$ junction.

Assuming this model of the reverse current, it is possible to assert that the increase of the reverse current 


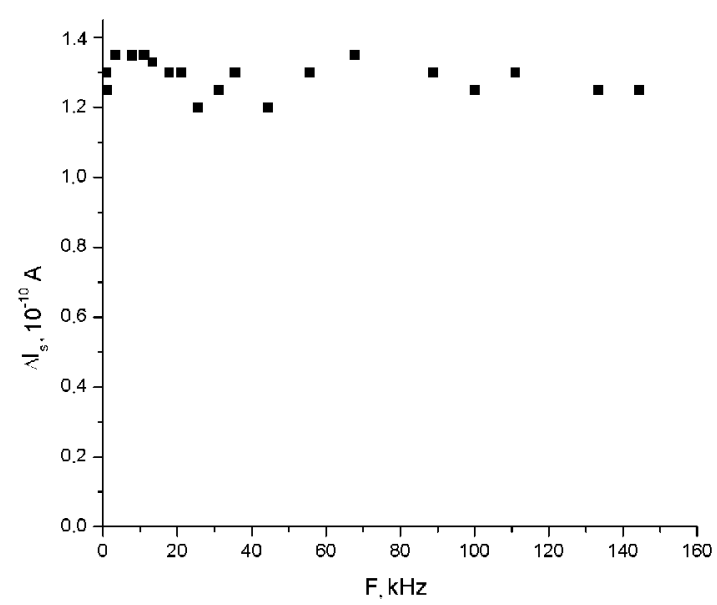

Fig. 4. Experimental data for the dependence of $\Delta I_{s}$ on the frequency of UHF modulation.

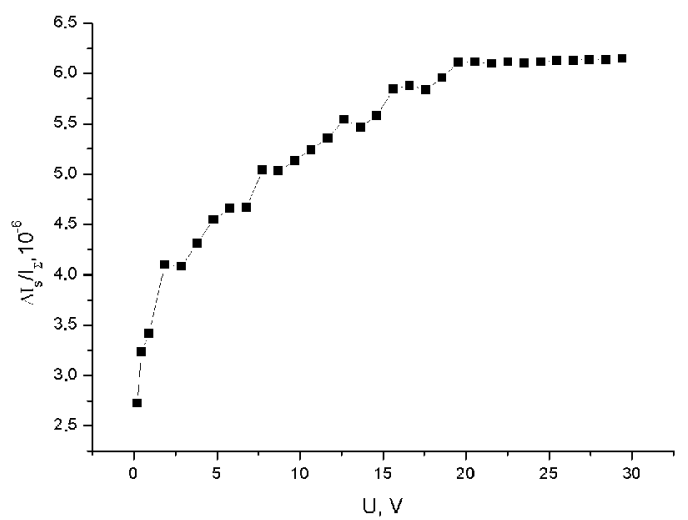

Fig. 5. Dependence of $\Delta I_{s} / I_{\Sigma}$ on the voltage.

under spin resonance conditions can be related with increasing the space charge of paramagnetic centres at the surface. The result of this - increase of the bending of energetic bands and growth of conductivity - was observed in our experiments. The dependence of $\Delta I_{s} / I_{\Sigma}$ on $U$ (Fig. 5) confirms this model. As an additional evidence for the proposed model, considered can be the fact of decreasing the effect's value under illumination of the diode polished side with strongly absorbed light.
The proposed mechanism of spin-dependent current in the silicon diode with $p$ - $n$ junction with polished sides (that includes $p-n$ junction) is the main one in our case. But this fact does not except involving the well known spin-dependent generation-recombination mechanism. The value of $I_{s}$ still remains unclear, but on the base of the obtained experimental results we offer the new model of spin-dependent current in diode, which matches qualitatively with experimental data.

\section{References}

1. G. Toth, Collision dependent du spin entre electrons de conduction et impurete's paramagnetiques dans les metaux et semiconductors: Effects sup les proprietes de transport. - The Doctorat Etat EsSciences Physiques, Paris, 1972, p.109.

2. D. Lepin, Spin-dependent recombination in silicon // Phys. Rev. B 6(2), p. 436-444 (1972).

3. L.S. Mima, V.I. Strikha, O.V. Tretyak // Fizika tekhnika poluprov. 14, p. 1328 (1980), in Russian.

4. L.S. Mima, O.V. Tretyak, Spin-dependent recombination in semiconductors // Fizika tekhnika poluprov. 15(9), p. 1729-1732 (1981), in Russian.

5. V.V. Ilchenko, O.V. Tretyak, Charge transport and spin-dependent recombination in polycrystalline silicon // Vestnik Kievskogo universiteta, ser. fizika, 1983, p. 24 (in Russian).

6. V.S. Lvov, L.S. Mima, O.V. Tretyak, Investigation of spin-dependent recombination in semiconductors // Preprint, vol. 182, Institute of Automation and Electrometry, Siberian Branch of Russian Academy of Sciences, Novosibirsk, Russia, 1982, p. 23 (in Russian).

7. I.M. Vikulin, V.I. Stafeyev, Physics of Semiconductor Devices. Radio i Svyaz Publ., Moscow, 1990, p. 40 (in Russian).

8. S.M. Ryvkin, Photoelectric Processes in Semiconductors. Gos. izd-vo fiz. mat. lit., Moscow, 1963, p. 60 (in Russian).

9. V.V. Pasinkov, L.K. Chirkin, A.D. Shinkov, Semiconductors Devices. Vysshaya Shkola Publ., Moscow, 1966, p. 122 (in Russian).

10. T.Ya. Gorbach, R.Yu. Holiney, I.M. Matiyuk et al., Electroreflectance spectroscopy and scanning electron microscopy study of microrelief silicon wafers with various surface pretreatments // Semiconductor Physics, Quantum Electronics \& Optoelectronics 1(1), p. 66-70 (1998). 